Research Article

\title{
Bipolar Magnetic and Thermospin Transport Properties of Graphene Nanoribbons with Zigzag and Klein Edges
}

\author{
Xingyi Tan $\mathbb{D}^{1},{ }^{1,2}$ Gang Xu $\left(\mathbb{D},{ }^{1}\right.$ Youchang Jiang, ${ }^{1}$ and Dahua Ren ${ }^{2}$ \\ ${ }^{1}$ Department of Physics, Chongqing Three Gorges University, Wanzhou 404100, China \\ ${ }^{2}$ School of Information Engineering, Hubei Minzu University, Enshi City 445000, China \\ Correspondence should be addressed to Xingyi Tan; tanxy@sanxiau.edu.cn
}

Received 9 September 2021; Revised 18 November 2021; Accepted 25 November 2021; Published 6 December 2021

Academic Editor: Georgios I. Giannopoulos

Copyright (c) 2021 Xingyi Tan et al. This is an open access article distributed under the Creative Commons Attribution License, which permits unrestricted use, distribution, and reproduction in any medium, provided the original work is properly cited.

Magnetic nanoribbons based on one-dimensional materials are potential candidates for spin caloritronics devices. Here, we constructed ferromagnetic graphene nanoribbons with zigzag and Klein edges $(N$-ZKGNRs, $N=4-21)$ and found that the $N$ ZKGNRs are in the indirect-gap bipolar magnetic semiconducting state (BMS). Moreover, when a temperature difference is applied through the nanoribbons, spin-dependent currents with opposite flow directions and opposite spin directions are generated, indicating the occurrence of the spin-dependent Seebeck effect (SDSE). In addition, the spin-dependent Seebeck diode effect (SDSD) also appeared in these devices. More importantly, we found that the BMS with a larger bandgap is promising for generating the SDSD, while the BMS with a smaller bandgap is promising for generating the SDSE. These findings show that ZKGNRs are promising candidates for spin caloritronics devices.

\section{Introduction}

Spin caloritronics, which focuses on the interaction between the spin, charge, and heat in materials, has attracted intensive interest because it plays an important role in the development of fundamental science and novel low-powerconsumption technologies [1-6]. In this field, an important effect is the spin-dependent Seebeck effect (SDSE) [7-9], which features different Seebeck thermopowers for different spins in spin-polarized systems. In recent years, there have been many reports concerning the SDSE in graphene nanoribbons (GNRs) with armchair or zigzag edges $[3,10,11]$. The original work was done by Zeng et al. [3], and they demonstrated the SDSE in magnetized zigzag GNRs (ZGNRs). Ni et al. [10] then proposed a new spin caloritronics device based on the ZGNR heterojunction and observed the SDSE. In previous work, we also observed the SDSE in GNRs and silicene nanoribbons (SiNRs) with armchair or zigzag edges [12-14]. As we have known, armchair and zigzag/Klein edges can be formed by cutting the graphene honeycomb lattice along the $<1 \overline{1} 10>$ and $<2$ $\overline{1} \overline{1} 0>$ directions, respectively. However, studies of the SDSE in ZGNRs with reconstructed edges (i.e., the Klein edge) and the pentagon-heptagon edge are rare. Actually, researchers have already experimentally observed these two types of reconstructed edges for zigzag GNRs $[15,16]$, and they have theoretically demonstrated that these edge reconstructions have drastic influences on the band structures and magnetic states of ZGNRs $[17,18]$. Hence, in this study, we investigated systematically the electronic structures and thermal spin-dependent transport characteristic of ZKGNRs by $a b$ initio calculations combined with the nonequilibrium Green's function approach. We found that ZKGNRs can be used in the stable bipolar magnetic semiconductor [19-21], in which the valence bands and conduction bands approach the Fermi level through opposite spin channels, and the SDSE can also be obtained. The results indicate that ZKGNRs are promising for application in spin caloritronics devices.

\section{Calculation Method and Model}

Here, we briefly introduce the device designs and theoretical method. The $N$-ZKGNRs in the present designs have one 
zigzag edge and one Klein edge (see Figures 1(a) and 1(b)), and both edges are saturated by two $\mathrm{H}$ atoms. Here, $N$ denotes the number of carbon rows across the GNR width, and $N=4-21$. We then built two-probe spin caloritronics devices based on the $N$-ZKGNRs ( $N=4$ as an example), as shown in Figure 1(c). The left and right contacts are semiinfinite ZKGNRs, and the central scattering region contains five units of ZKGNRs. We focus on the spin currents driven by the temperature difference $(\Delta T)$ between the source temperature $T_{L}$ and drain temperature $T_{R}$, that is, $\Delta T=T_{L}-T_{R}$.

All of the calculations were performed with the Atomistix Toolkit (ATK) package [22, 23], which uses spin density functional theory in combination with the nonequilibrium Green's function method. The geometry optimization and electronic structure calculations were performed with the double-zeta-polarized (DZP) basis set, and the exchange-correlation potential was treated by the generalized gradient approximation method [24, 25]. The cutoff energy was 75 hartree, and a Monkhorst-Pack $1 \times 1 \times 100 \mathrm{k}$-mesh was chosen. In the Landauer-Büttiker formalism, the spin-dependent currents of the devices were obtained with the following equation [26]:

$$
I^{\uparrow(\downarrow)}=\frac{e}{h} \int_{-\infty}^{\infty}\left\{T^{\uparrow(\downarrow)}(E)\left[f_{L}\left(E, T_{L}\right)-f_{R}\left(E, T_{R}\right)\right]\right\} \mathrm{d} E,
$$

where $e$ is the electron charge, $h$ is the Planck constant, and $T_{L(R)}$ is the temperature of the left (right) electrode. $f_{L(R)}$ $\left(E, T_{L(R)}\right)$ is the average Fermi-Dirac distribution of the left (right) electrode:

$$
f_{L(R)}\left(E, T_{L(R)}\right)=\left\{1+\exp \left[\frac{\left(E-\mu_{L(R)}\right)}{k_{B} T_{L(R)}}\right]\right\}^{-1},
$$

where $\mu_{(R)}$ and $T_{L(R)}$ are the chemical potential and temperature of the left (right) electrode, respectively, and $k_{B}$ is the Boltzmann constant. $T^{\uparrow(\downarrow)}(E)$ is the spin-dependent transport coefficient:

$$
T^{\uparrow(\downarrow)}(E)=\operatorname{Tr}\left[\Gamma_{L} G^{r} \Gamma_{R} G^{a}\right],
$$

where $G^{r(a)}$ is Green's function that is retarded in the central region and $\Gamma_{L(R)}$ is the coupling matrix for the left (right) electrode. In addition, the calculation methods of the spinup thermopower $\left(S_{u p}\right)$, spin-down thermopower $\left(S_{d n}\right)$, total charge thermopower $\left(\mathrm{S}_{\mathrm{ch}}\right)$, and net spin thermopower $\left(\mathrm{S}_{\mathrm{sp}}\right)$ can be found in our previous work [14].

\section{Results and Discussion}

First, we demonstrate the magnetic ordering at the edges of the $N$-ZKGNRs $(N=4-21)$ (Figure 2). To this end, we consider the total energies of three different magnetic phases, i.e., ferromagnetic (FM), antiferromagnetic (AFM), and nonmagnetic (NM) ordering, between two edges in the nanoribbons. The energy differences $\mathrm{E}_{\mathrm{FM}-\mathrm{AFM}}=\mathrm{E}_{\mathrm{FM}}-\mathrm{E}_{\mathrm{AFM}}$ and $E_{\mathrm{FM}-\mathrm{NM}}=\mathrm{E}_{\mathrm{FM}}-\mathrm{E}_{\mathrm{NM}}$, where $\mathrm{E}_{\mathrm{FM}}, \mathrm{E}_{\mathrm{AFM}}$, and $\mathrm{E}_{\mathrm{NM}}$ stand for the total energies of FM, AFM, and NM ordering between two edges in the nanoribbons, are shown in Figure 2. It is obvious that all the $\mathrm{E}_{\mathrm{FM}-\mathrm{AFM}}$ and $\mathrm{E}_{\mathrm{FM}-\mathrm{NM}}$ are less than zero, confirming the FM ground state in the $N$ ZKGNRs.

Next, we investigated the band structures of the $N$ ZKGNRs $(N=4-21)$ (Figure 3$)$. The band structure of the nanoribbons noticeably changes as $N$ increases. For $N=4$ and 5, the conduction band minimum (CBM) states are related to the spin-down states and located at the $\Gamma$ point, which are above the Fermi level $\left(E_{F}\right)$, and the spin-up states below $E_{F}$ serve as the valence band maximum (VBM) states and are located at the $Z$ point. The valence and conduction bands possess opposite spin polarization as they approach $E_{F}$. Moreover, the spin-dependent bands of these ZKGNRs have finite gaps around $E_{F}$.

These characteristics indicate that 4-ZKGNR and 5ZKGNR are indirect-gap BMSs (IBMSs) [27-29]. As the nanoribbon width increases, the ferromagnetic configuration is maintained, whereas the band structure near the Fermi level remarkably changes. For $N=6$, the CBM is related to the spindown states and located at the $\Gamma$ point, and the spin-up states below $E_{F}$ serve as the VBM and are located at the $\Gamma-Z$ line. These characteristics indicate that $6-\mathrm{ZKGNR}$ is also an indirect-gap BMS. For $N=7-21$, both the VBM of the spin-up channel and the CBM of the spin-down channel are located at the $\Gamma-Z$ line with the same point. These characteristics indicate that these ZKGNRs are direct-gap BMSs (DBMSs). The corresponding bandgaps are shown in Figure 4. In summary, as the width increases, the ZKGNR may have two different states, that is, the IBMS state and the DBMS state (Figure 4).

To explore the SDSE, we turn our attention to the ZKGNR thermal spin transport properties. The spin-dependent currents through the $N$-ZKGNR (taking $N=4$ and 21 as examples) devices versus $T_{L}$ and $\Delta T$ are shown in Figure 5. For the 4-ZKGNR device, there are no spin-up currents $\left(I_{u p}\right)$ and spin-down currents $\left(I_{d n}\right)$ when $T_{L}<300 \mathrm{~K}$ for the three values of $\Delta T$. This suggests that no thermalinduced spin-dependent currents are generated in this $T_{L}$ range, irrespective of the temperature difference $(\Delta T)$. In other words, there is a threshold temperature $\left(T_{t h}\right)$ at approximately $300 \mathrm{~K}$ for $I_{u p}$ and $I_{d n}$. When $T_{L}>T_{t h}$, both $I_{u p}$ and $I_{d n}$ sharply increase with increasing $T_{L}$. However, they flow in opposite directions, that is, $I_{u p}$ is negative and $I_{d n}$ is positive. There is no doubt that this is caused by the SDSE [30-34]. Furthermore, the spin-dependent current is larger for higher $\Delta T$. The curves of spin-dependent current versus $\Delta T$ are shown in Figure $5(\mathrm{~b})$, with $T_{L}$ set to 300,350 , and $400 \mathrm{~K}$. For $\Delta T>0$, the curves clearly indicate that the spindependent currents are approximately symmetric about the zero-current axis and robust over a large range of temperature difference. Therefore, the SDSE can also be confirmed by the curves of spin-dependent current versus $\Delta T$. For $\Delta T<0$, both $I_{u p}$ and $I_{d n}$ are approximately equal to zero. These characteristics indicate that the perfect SDSD occurs in the 4-ZKGNR device. The 21-ZKGNR device has similar characteristics to the 4-ZKGNR device, but it has smaller $T_{t h}$ $\left(T_{t h}=80 \mathrm{~K}\right)$ and larger $I_{u p}$ and $I_{d n}$, as shown in Figure 5(c). In addition, as shown in Figure 5(d), both $I_{u p}$ and $I_{d n}$ of the 21ZKGNR are larger than zero, indicating a weak SDSD. 


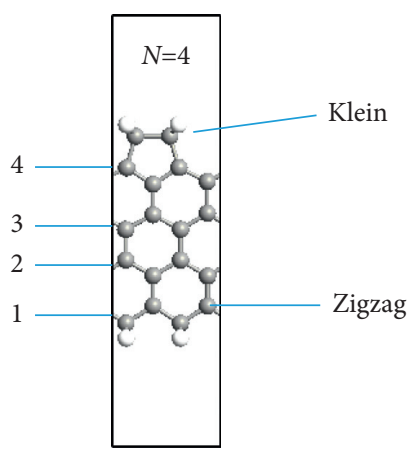

(a)

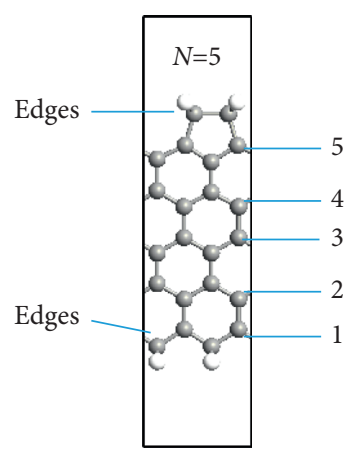

(b)

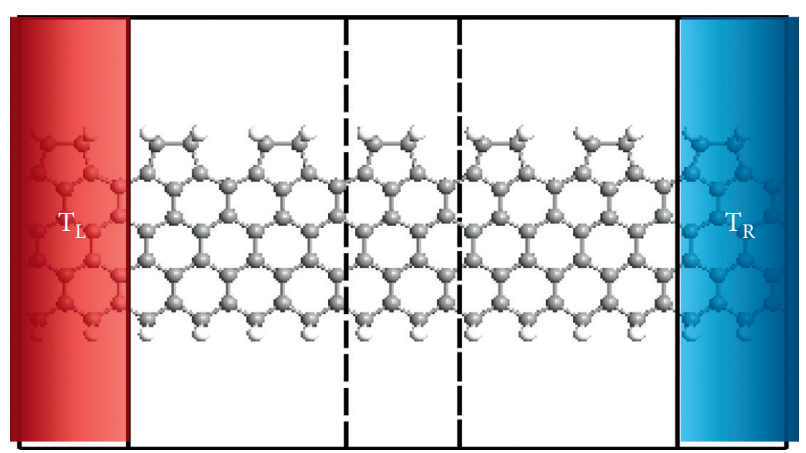

(c)

Figure 1: (a, b) Optimal structure of the N-ZKGNRs (say $N=4,5$ ), in which carbon and hydrogen atoms are described by the silver and white balls, respectively. (c) Schematic description of the thermal spin device using N-ZKGNRs (say $N=4$ ).

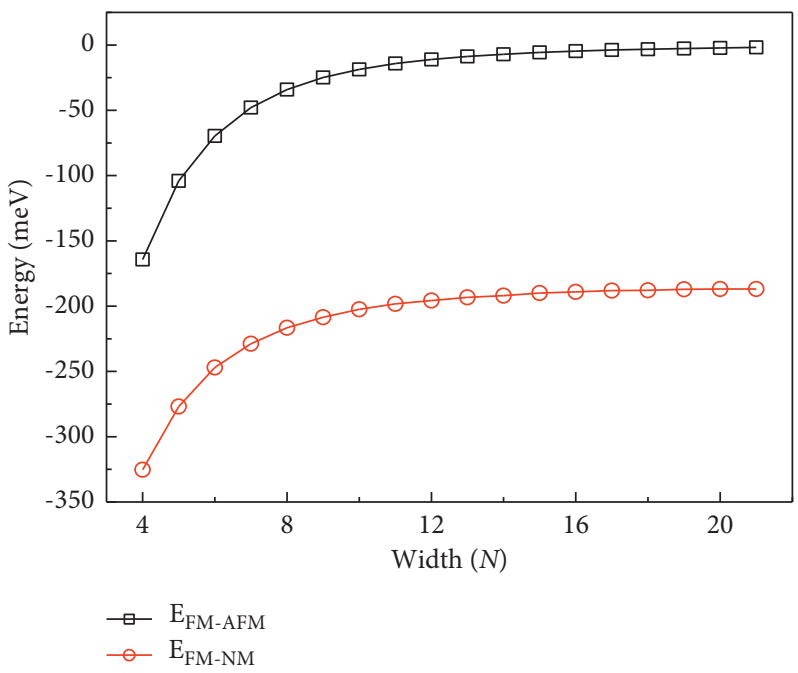

Figure 2: The energy difference $\mathrm{E}_{\mathrm{FM}-\mathrm{AFM}}$ and $\mathrm{E}_{\mathrm{FM}-\mathrm{NM}}$ of the N-ZKGNRs $(N=4-21)$.
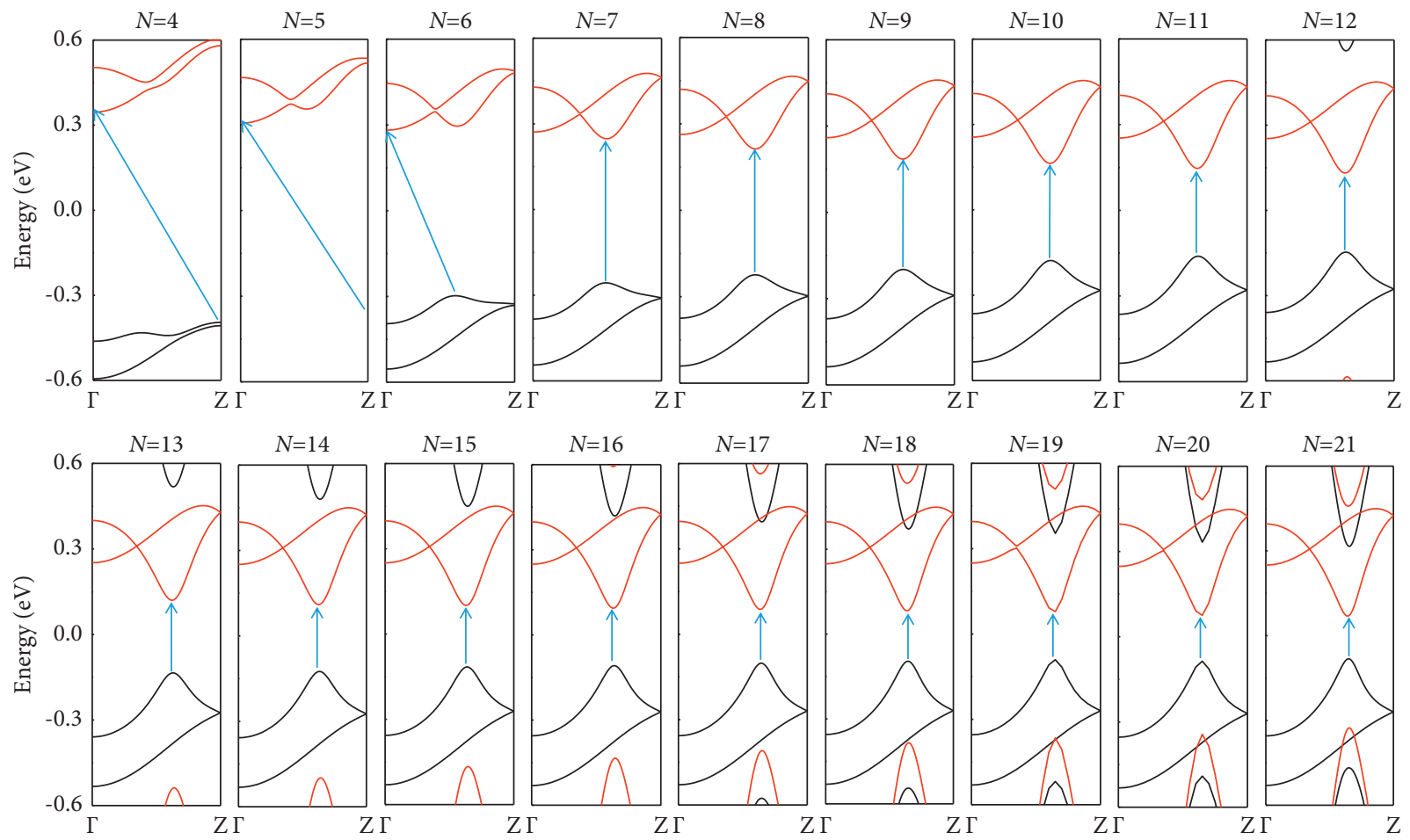

FIGURE 3: Spin-dependent band structures of the N-ZKGNRs $(N=4-21)$. The black and red lines represent spin-up and spin-down electrons, respectively. 


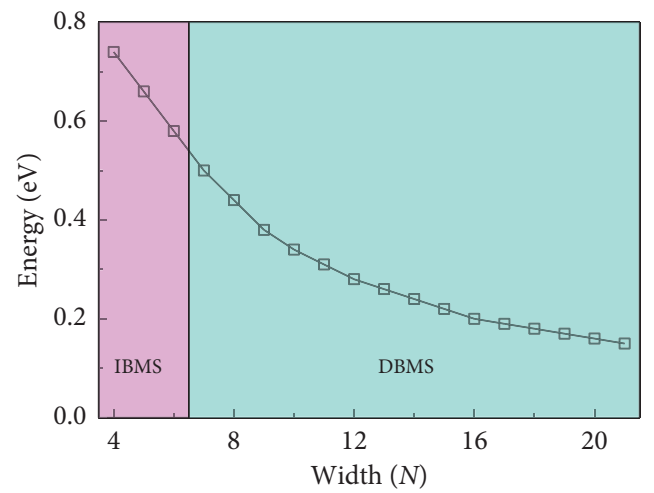

FIgURE 4: Change in the bandgap energy in terms of the width of the N-ZKGNRs $(N=4-21)$. The IBMS and DBMS represent indirect-gap bipolar magnetic semiconductor and direct-gap bipolar magnetic semiconductor, respectively.

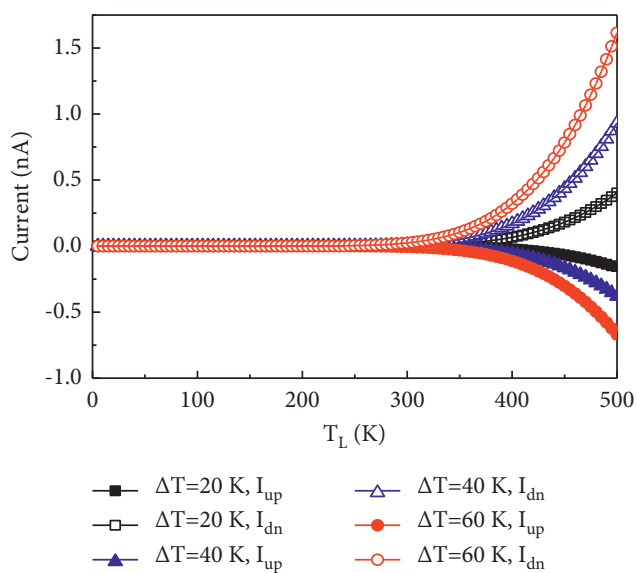

(a)

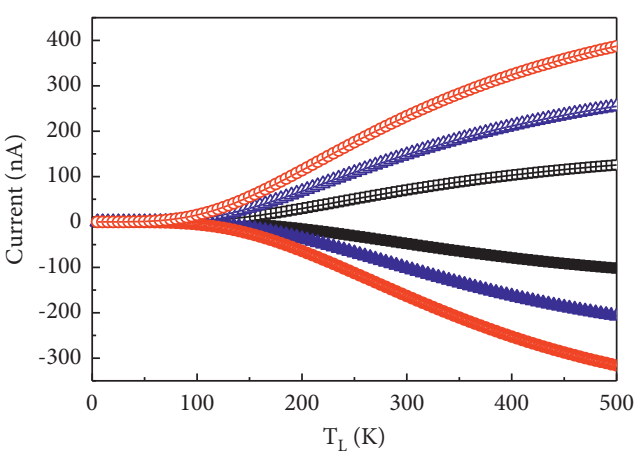

(c)

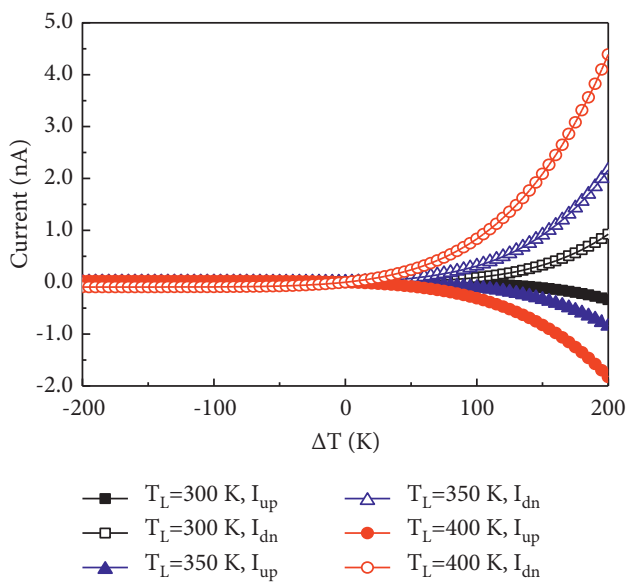

(b)

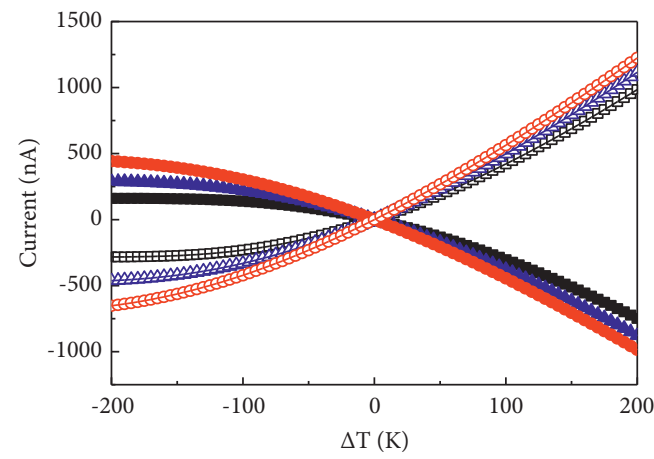

(d)

FIGURE 5: Spin-up currents $\left(I_{u p}\right)$ and spin-down currents $\left(I_{d n}\right)$ in terms of $T_{L}$ for the N-ZKGNRs devices at selected temperature difference: (a) $N=4$; (c) $N=21$. $I_{u p}$ and $I_{d n}$ in terms of $\Delta T$ for selected values of $T_{L}$ : (b) $N=4$; (d) $N=21$.

To illustrate the underlying mechanism of these phenomena, we plot the corresponding spin-dependent transmissions of the $N$-ZKGNRs $(N=4,21)$ in Figure 6 . For the 4-ZKGNR (Figure 6(a)), the spin-up and spin-down transmissions are nearly symmetrically localized downside and upside of the Fermi level, which provides two independent spin-conducting channels. If a temperature difference is applied along the nanoribbons, the spin-down and spin-up current have opposite flow directions, unambiguously representing the occurrence of the SDSE, as shown in Figures 5(a) and 5(b). For the 21-ZKGNR (Figure 6(b)), the spin-dependent transmissions have similar characteristics to the 4-ZKGNR, except for a smaller bandgap, resulting in the smaller $T_{t h}$, as shown in Figure 5(c). Therefore, the SDSE also occurs. It is important that we found that $T_{t h}$ has a positive correlation with the 


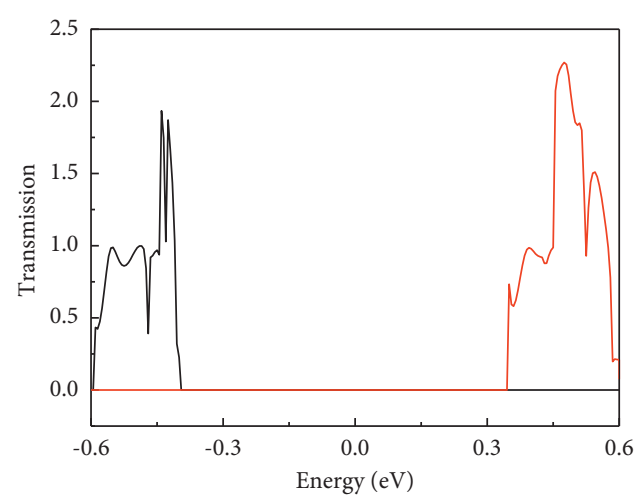

(a)

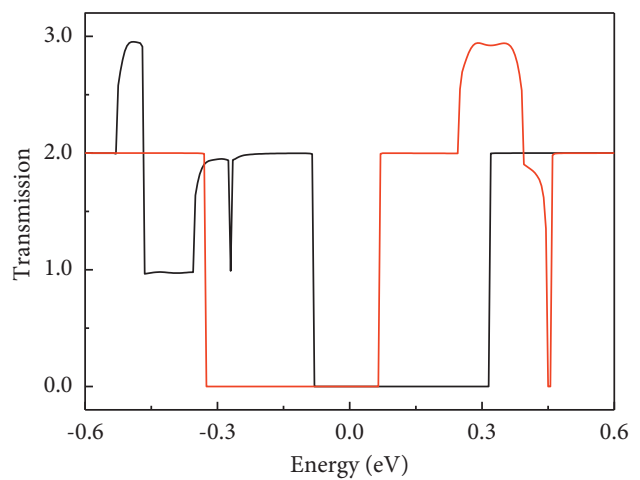

(b)

Figure 6: Spin-related transmission spectrums of the N-ZKGNRs devices: (a) $N=4$; (b) $N=21$, in which spin-up and spin-down transmissions are described by the solid black and red lines.

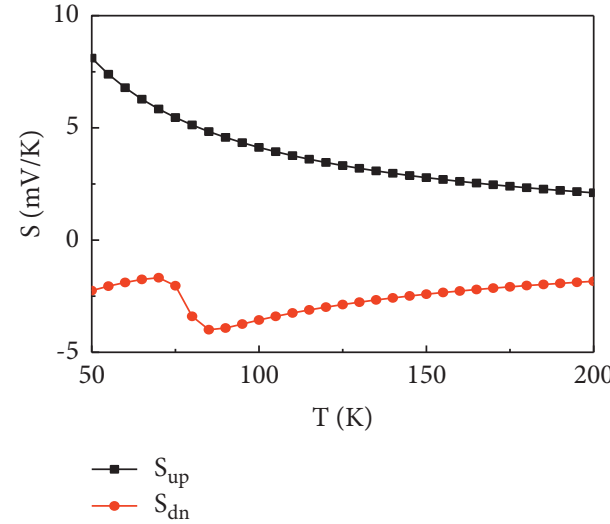

(a)

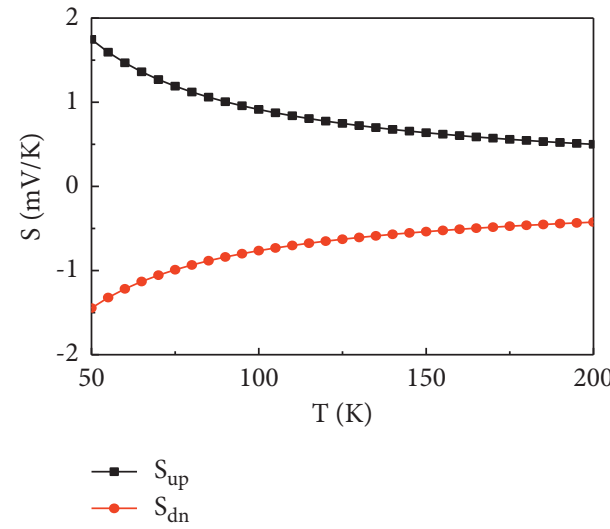

(c)

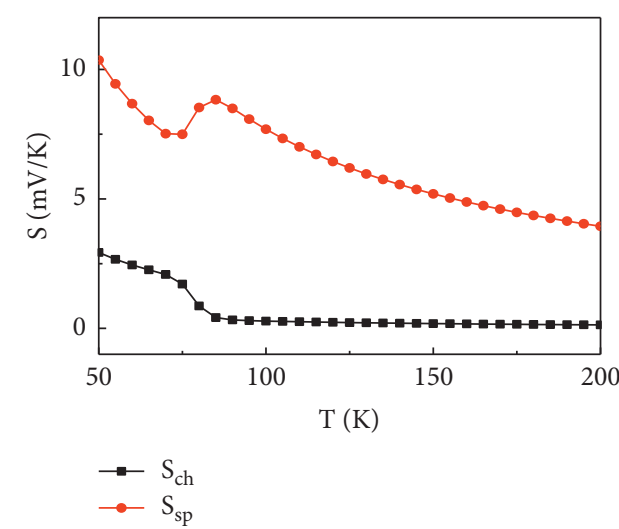

(b)

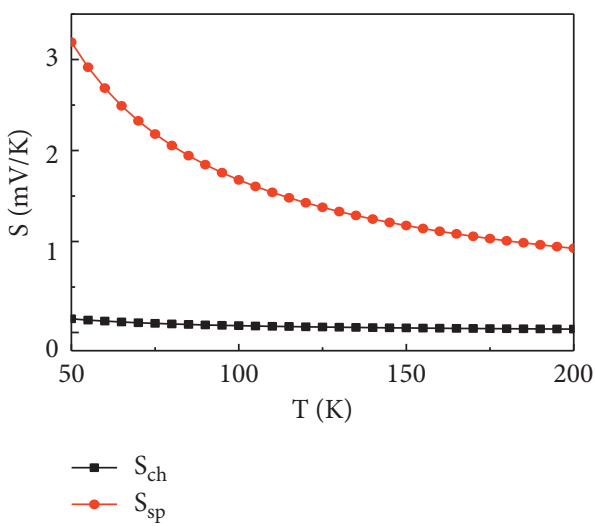

(d)

FiguRE 7: The spin-dependent Seebeck coefficients $S_{\text {up }}$ and $S_{\text {dn }}$, charge Seebeck coefficient $S_{\text {ch }}$, and spin Seebeck coefficient $S_{\text {sp }}$ as a function of device temperature $\mathrm{T}$ at the zero chemical potential $(\mu=0)$. (a, b) Results of 4-ZKGNR; (c, d) results of 21-ZKGNR.

bandgap, that is, $T_{t h}$ is larger for larger bandgap. Therefore, we can draw the following conclusion: the BMSs with larger bandgaps are promising candidates for generating the SDSD, while the BMSs with smaller bandgaps are promising candidates for generating the SDSE.
For further in-depth study into the spin thermal transport, we calculated the spin-up thermopower $\left(S_{u p}\right)$, spin-down thermopower $\left(S_{\mathrm{dn}}\right)$, total charge thermopower $\left(\mathrm{S}_{\mathrm{ch}}\right)$, and net spin thermopower $\left(\mathrm{S}_{\mathrm{sp}}\right)$ versus the device temperature at the Fermi level $\left(E_{F}=0\right)$. As shown in 
Figure 7, $S_{\mathrm{up}}>0$, while $S_{\mathrm{dn}}<0$, supporting the appearance of SDSE in these two devices. In particular, the $S_{\mathrm{ch}}$ of 21ZKGNR is nearly equal to zero, indicating a perfect SDSE. Furthermore, the $S_{\text {sp }}$ of 4-ZKGNR is larger than the $S_{\text {sp }}$ of 21-ZKGNR because 4-ZKGNR has a larger band gap.

\section{Conclusion}

We have investigated the electronic structures and thermal spin-dependent transport properties of a series of $\mathrm{N}$ ZKGNRs by first-principle calculations combined with the nonequilibrium Green's function. First, we found that as the nanoribbon width parameter $N$ is increased from 4 to 21 , the $\mathrm{N}$-ZKGNR transforms from an indirect-gap BMS to a directgap BMS. Second, the SDSE and SDSD appear if we produce a temperature difference through these ZKGNRs. Finally, the BMSs with larger bandgaps are promising candidates for generating the SDSD, while the BMSs with smaller bandgaps are promising candidates for generating the SDSE. These findings strongly suggest that $N$-ZKGNRs are promising materials for spin caloritronics devices.

\section{Data Availability}

Data are available upon request.

\section{Conflicts of Interest}

The authors declare no conflicts of interest.

\section{Acknowledgments}

This work was supported by the National Natural Science Foundation of China (grant no. 11864011), in part by the Science and Technology Research Program of Chongqing Municipal Education Commission (KJQN202001207 and KJQN202101204), and in part by the Educational Commission of Hubei Province of China (T201914). The authors thank Liwen Bianji (Edanz) (http://www.liwenbianji.cn/) for editing the English text of a draft of this manuscript.

\section{References}

[1] C. M. Jaworski, J. Yang, S. Mack, D. D. Awschalom, J. P. Heremans, and R. C. Myers, "Observation of the spinSeebeck effect in a ferromagnetic semiconductor," Nature Materials, vol. 9, no. 11, pp. 898-903, 2010.

[2] K. Uchida, J. Xiao, H. Adachi et al., "Spin Seebeck insulator," Nature Materials, vol. 9, no. 11, pp. 894-897, 2010.

[3] M. Zeng, Y. Feng, and G. Liang, "Graphene-based spin caloritronics," Nano Letters, vol. 11, no. 3, pp. 1369-1373, 2011.

[4] G. E. W. Bauer, E. Saitoh, and B. J. Van Wees, "Spin caloritronics," Nature Materials, vol. 11, no. 5, pp. 391-399, 2012.

[5] S. R. Boona, R. C. Myers, and J. P. Heremans, "Spin caloritronics," Energy \& Environmental Science, vol. 7, no. 3, pp. 885-910, 2014.

[6] H. H. Fu and K.-L. Yao, "Perfect thermal spin filter and pure spin thermoelectric generator based on a laterally coupled double quantum-dot array," EPL (Europhysics Letters), vol. 103, no. 5, Article ID 57011, 2013.
[7] A. Slachter, F. L. Bakker, J.-P. Adam, and B. J. van Wees, "Thermally driven spin injection from a ferromagnet into a non-magnetic metal," Nature Physics, vol. 6, no. 11, pp. 879-882, 2010.

[8] J.-C. Le Breton, S. Sharma, H. Saito, S. Yuasa, and R. Jansen, "Thermal spin current from a ferromagnet to silicon by Seebeck spin tunnelling," Nature, vol. 475, no. 7354, pp. 82-85, 2011.

[9] B. Z. Rameshti and A. G. Moghaddam, "Spin-dependent Seebeck effect and spin caloritronics in magnetic graphene," Physical Review B, vol. 91, no. 15, Article ID 155407, 2015.

[10] Y. Ni, K. Yao, H. Fu, G. Gao, S. Zhu, and S. Wang, "Spin Seebeck effect and thermal colossal magnetoresistance in graphene nanoribbon heterojunction," Scientific Reports, vol. 3, no. 1, Article ID 1380, 2013.

[11] D.-D. Wu and H.-H. Fu, "Spin-Seebeck effect and thermal colossal magnetoresistance in the narrowest zigzag graphene nanoribbons," Nanotechnology, vol. 32, no. 24, Article ID 245703, 2021.

[12] X.-Q. Tang, X.-M. Ye, X.-Y. Tan, and D.-H. Ren, "Metal-free magnetism, spin-dependent Seebeck effect, and spin-Seebeck diode effect in armchair graphene nanoribbons," Scientific Reports, vol. 8, no. 1, p. 927, 2018.

[13] X.-Y. Tan, D.-D. Wu, Q.-B. Liu, H.-H. Fu, and R. Wu, "Spin caloritronics in armchair silicene nanoribbons with sp 3 and sp 2-type alternating hybridizations," Journal of Physics: Condensed Matter, vol. 30, no. 35, Article ID 355303, 2018.

[14] X. Tan, L. Liu, G.-F. Du, and H.-H. Fu, "Thermal transport and spin-dependent Seebeck effect in parallel step-like zigzag graphene nanoribbon junctions," Physical Chemistry Chemical Physics, vol. 22, no. 34, pp. 19100-19107, 2020.

[15] C. O. Girit, J. C. Meyer, R. Erni et al., "Graphene at the edge: stability and dynamics," Science, vol. 323, no. 5922, pp. 1705-1708, 2009.

[16] K. Suenaga and M. Koshino, "Atom-by-atom spectroscopy at graphene edge,” Nature, vol. 468, no. 7327, pp. 1088-1090, 2010.

[17] P. Koskinen, S. Malola, and H. Häkkinen, "Self-passivating edge reconstructions of graphene," Physical Review Letters, vol. 101, no. 11, Article ID 115502, 2008.

[18] P. Wagner, C. P. Ewels, J.-J. Adjizian et al., "Band gap engineering via edge-functionalization of graphene nanoribbons," Journal of Physical Chemistry C, vol. 117, no. 50, pp. 26790-26796, 2013.

[19] X. Li, X. Wu, Z. Li, J. Yang, and J. G. Hou, "Bipolar magnetic semiconductors: a new class of spintronics materials," Nanoscale, vol. 4, no. 18, pp. 5680-5685, 2012.

[20] Z. F. Wang, S. Jin, and F. Liu, "Spatially separated spin carriers in spin-semiconducting graphene nanoribbons," Physical Review Letters, vol. 111, Article ID 096803, 2013.

[21] X. Li and J. Yang, "First-principles design of spintronics materials," National Science Review, vol. 3, no. 3, pp. 365-381, 2016.

[22] M. Brandbyge, J.-L. Mozos, P. Ordejón, J. Taylor, and K. Stokbro, "Density-functional method for nonequilibrium electron transport," Physical Review B, vol. 65, no. 16, Article ID 165401, 2002.

[23] S. Smidstrup, T. Markussen, and P. Vancraeyveld, "QuantumATK: an integrated platform of electronic and atomicscale modelling tools," Journal of Physics: Condensed Matter, vol. 32, Article ID 015901, 2020.

[24] J. P. Perdew and Y. Wang, "Accurate and simple analytic representation of the electron-gas correlation energy," Physical Review B, vol. 45, no. 23, pp. 13244-13249, 1992. 
[25] J. P. Perdew, K. Burke, and M. Ernzerhof, "Generalized gradient approximation made simple," Physical Review Letters, vol. 77, no. 18, pp. 3865-3868, 1996.

[26] Y. Imry and R. Landauer, "Conductance viewed as transmission," Reviews of Modern Physics, vol. 71, no. 2, pp. S306-S312, 1999.

[27] X. Tan, X. Xu, L. Ding, and Y. He, "Linear and nonlinear thermal spin transport properties of zigzag $\alpha$-graphyne nanoribbons with sp-sp edges," Chemical Physics Letters, vol. 777, Article ID 138724, 2021.

[28] X. Tan, L. Zhang, and L. Liu, "Bipolar magnetic semiconductor properties and spin-dependent Seebeck effects induced by nanoscale graphene domains doped into armchair boron nitride nanoribbons," Chemical Physics Letters, vol. 748, Article ID 137386, 2020.

[29] J. Zhang, X. Li, and J. Yang, "SiN-SiC nanofilm: a nanofunctional ceramic with bipolar magnetic semiconducting character," Applied Physics Letters, vol. 104, no. 17, Article ID 172403, 2014.

[30] H.-H. Fu, D.-D. Wu, L. Gu, M. Wu, and R. Wu, "Design for a spin-Seebeck diode based on two-dimensional materials," Physical Review B, vol. 92, Article ID 045418, 2015.

[31] H.-H. Fu, D.-D. Wu, Z.-Q. Zhang, and L. Gu, "Spin-dependent Seebeck effect, thermal colossal magnetoresistance and negative differential thermoelectric resistance in zigzag silicene nanoribbon heterojunciton," Scientific Reports, vol. 5, no. 1, Article ID 10547, 2015.

[32] H.-H. Fu, L. Gu, and D.-D. Wu, "A spin-Seebeck diode with a negative differential spin-Seebeck effect in a hydrogen-terminated zigzag silicene nanoribbon heterojunction," Physical Chemistry Chemical Physics, vol. 18, no. 18, pp. 12742-12747, 2016.

[33] Z.-Q. Zhang, Y.-R. Yang, H.-H. Fu, and R. Wu, "Design of spin-Seebeck diode with spin semiconductors," Nanotechnology, vol. 27, no. 50, Article ID 505201, 2016.

[34] D.-D. Wu, Q.-B. Liu, H.-H. Fu, and R. Wu, "How to realize a spin-dependent Seebeck diode effect in metallic zigzag $\gamma$-graphyne nanoribbons?" Nanoscale, vol. 9, no. 46, pp. 18334-18342, 2017. 